\title{
Emergencia de la cepa W135 causante de enfermedad meningocócica invasora en Chile 2012
}

\author{
M. TERESA VALENZUELA ${ }^{1}$, GABRIELA MORENO $^{2}$, ALEJANDRA VAQUERO $^{3, \mathrm{a}}$, \\ MABEL SEOANE ${ }^{4, b}$, J. CARLOS HORMAZÁBAL ${ }^{5}$, M. PAZ BERTOGLIA ${ }^{3, c}$, \\ DORIS GALLEGOS ${ }^{6, \mathrm{~d}}$, VIVIANA SOTOMAYOR ${ }^{6, \mathrm{~d}}$, JORGE DÍAZ7
}

\section{Emergence of W135 meningococcal serogroup in Chile during 2012}

The epidemiologic behavior of the Invasive Meningococcal Disease (IMD) in Chile has changed. At the end of 2011, the W135 serogroup belonging to the hypervirulent clone ST-11 emerged. It affected diverse countries of the world, after the Mecca pilgrimage in 2000. In Chile, there have been 133 IMD cases during 2012. These figures represent an incidence of 0.7 per 100,000 inhabitants, which is 30\% higher than expected. Eighty eight percent of cases were confirmed by the National Reference Laboratory at the Chilean Public Health Institute. The serogroup was determined in 103 strains and 58\% belonged to the W135 serogroup, surpassing for the first time the B serogroup (37\%). The Metropolitan Region concentrated $80 \%$ of these cases, and the remaining $20 \%$ affected other seven regions of the country. Forty seven percent of cases corresponded to children less than 5 years of age. The predominant clinical presentation of the W135 serogroup was a sepsis in $67 \%$ of cases. The fatality ratio of IDM during 2012 was 27\%, the highest in the past 20 years. With this information, the Chilean Ministry of Health decreed a sanitary alert and implemented an integrated approach to control and prevent W-135 IDM, denominated "W-135 Action Plan".

(Rev Med Chile 2013; 141: 959-967). Sepsis.

Key words: Epidemiological Monitoring; Neisseria meningitidis serogroup W135;

L a enfermedad meningocócica invasora (EMI) es una patología infecciosa grave causada por Neisseria meningitidis, un diplococo Gram (-). Las presentaciones clínicas comunes son meningitis, septicemia o meningococcemia y una combinación de ambas ${ }^{1}$.

La infección por Neisseria meningitidis (meningococo) afecta tanto a países desarrollados como en desarrollo, se presenta en forma endémica y epidémica y es un gran problema de salud pública a nivel mundial ${ }^{2}$. En los últimos 30 años, los brotes de mayor magnitud y frecuencia han ocurrido en África sub-sahariana. También se han presentado brotes con menor incidencia en Europa y las Américas ${ }^{3}$.

\author{
'Dirección Instituto de \\ Salud Pública de Chile. \\ ²División de Planificación \\ Sanitaria Ministerio de \\ Salud Chile. \\ ${ }^{3}$ Departamento de Asuntos \\ Científicos. Instituto de \\ Salud Pública de Chile. \\ ${ }^{4}$ Laboratorio de Referencia \\ de Meningitis. Instituto de \\ Salud Pública de Chile. \\ ${ }^{5}$ Subdepartamento \\ Enfermedades Infecciosas. \\ Instituto de Salud Pública \\ de Chile. \\ ${ }^{6}$ Departamento de \\ Epidemiología. Ministerio \\ de Salud Chile. \\ ${ }^{7}$ Subsecretaría de Salud \\ Pública. Ministerio de Salud \\ de Chile. \\ ${ }^{a}$ Médico Veterinario.

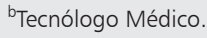 \\ 'Kinesióloga. \\ dEnfermera Universitaria. \\ Recibido el 11 de enero de \\ 2013, aceptado el 11 de \\ junio de 2013. \\ Correspondencia a: \\ M. Teresa Valenzuela \\ Marathon 1000, Ñuñoa, \\ Santiago \\ E-mail:mtvalenzuela@ \\ ispch.cl
}

Adicionalmente, el meningococo puede ser un comensal de la faringe humana, transmisible a contactos cercanos a través de gotas desde las secreciones respiratorias. Entre 4 y $35 \%$ de la población puede ser portadora en períodos interepidémicos. Los factores asociados a mayores tasas de portación son las infecciones respiratorias por influenza o micoplasma, exposición al tabaco, cocinar a leña, asistencia a bares y discotecas, consumo excesivo de alcohol. Las razones por las que algunas personas desarrollan la enfermedad se deben a interacciones complejas que involucran al hospedero y al agente ${ }^{5}$, dentro de los factores genéticos del huésped se encuentran las deficiencias en los factores terminales del complemento ${ }^{4}$. 
De acuerdo a los resultados de un estudio en Estados Unidos de Norteamérica entre 1994 y 2002, los brotes dan cuenta de menos de $2 \%$ del total de casos reportados, pero la tasa de letalidad es más alta durante éstos que en los períodos endémicos ${ }^{6}$. Las secuelas neurológicas que persisten a los 6 meses después del diagnóstico son; sordera, déficit locomotor, parálisis del nervio oculomotor, epilepsia, retraso del desarrollo psicomotor y cognitivo ${ }^{7}$.

\section{Antecedentes históricos}

En Chile se ha mantenido vigilancia epidemiológica y de laboratorio de Neisseria meningitidis desde $1976^{2}$, con incidencia de 0,2 a 2 por 100.000 habitantes ${ }^{8}$ y predominio endémico inicialmente del serogrupo C $(90 \%)^{2}$, siendo el serogrupo B poco frecuente 9 .

En 1978 se registró un brote en la Región Metropolitana que dio origen a un programa de vacunación masiva (vacuna A-C), con una cobertura de $95 \%$ al grupo entre 6 meses y 25 años, acompañado de un estudio serológico de la respuesta inmune cuya respuesta fue satisfactoria ${ }^{2}$. En el año 1982, comenzó a aumentar la incidencia del serogrupo $\mathrm{B}^{8}$.

Entre 1982 y 1992, las tasas de ataque evidenciaron un aumento de 17,3\% por año. En 1986, se presentó un brote en la zona norte del país, especialmente en Iquique, alcanzando tasas de 29,2 por 100.000 habitantes, las que comenzaron a declinar a partir de 1989 , con tasas de 19,5 por 100.000 habitantes$^{9}$. Entre los años 1992 y 1993, nuestro país se vio afectado por un nuevo brote, incrementando la tasa de incidencia a 3,8 por 100.000 habitantes y el serogrupo predominante fue el B (91,1\% y $94,7 \%$ respectivamente), seguido por el W135. Los serotipos y subtipos más frecuentemente asociados a este serogrupo fueron B:15:P1.3 y B:NT:P1.3 ${ }^{8}$.

Desde mediados de la década 1990-99, el serogrupo C pasa a representar $20 \%$ de los casos. La incidencia global de EMI se estabilizó alrededor de 3,7 casos por 100.000 habitantes, lo que representa aproximadamente 500 casos anuales. Existió un aumento en el año 1994, con una incidencia de 4,5 por 100.000 habitantes $^{2,9}$.

En el año 2000 comenzó un descenso de los casos hasta alcanzar en el 2006 una tasa de incidencia de 0,8 casos por 100.000 habitantes (alrededor de 120 notificaciones anuales). El grupo de edad más afectado son los menores de 5 años, especialmente, los menores de $1 \mathrm{año}^{2}$. En el año 2011 la enfermedad presentó una incidencia de 0,4 por 100.000 , representando la tasa más baja del período analizado. La letalidad presentó variaciones importantes en el último decenio con tendencia al alza, alcanzando $14,7 \%$ en el $2011^{10}$.

En el año 2012, debido al aumento de casos de EMI por serogrupo W135, el Ministerio de Salud en conjunto con otras instituciones públicas y privadas del país, apoyados por la Organización Panamericana de la Salud (OPS) y el Centers for Disease Control and Prevention (CDC) de Estados Unidos de Norteamérica, desarrolló una estrategia denominada "Plan de acción W-135" para abordar en forma integral el control y la prevención de la EMI W-135, la que incluyó la conformación del Comité Nacional W-135, refuerzo del sistema de vigilancia epidemiológica y de laboratorio, educación a la población y prevención a través de un programa de vacunación de la población de mayor riesgo.

El objetivo del presente artículo es dar a conocer el comportamiento epidemiológico, clínico y de laboratorio de los casos de EMI confirmados por el Laboratorio de Referencia como Neisseria meningitidis ocurridos en Chile durante el año 2012, comparando el comportamiento del serogrupo W135 con los demás.

\section{Material y Método}

\section{Sistema de vigilancia epidemiológica en Chile}

Chile es un país con una población de 16.572.475 habitantes al $2012^{11}$ y los menores de 5 años constituyen $7,2 \%{ }^{12}$.

El sistema de salud es mixto, sin embargo, la vigilancia de las enfermedades transmisibles es universal y de responsabilidad del Estado.

El Sistema de Vigilancia se encuentra establecido en el Decreto Supremo 158, del Ministerio de Salud (MINSAL) e incorpora a la EMI como una enfermedad de notificación obligatoria inmediata, bajo los códigos CIE10 A39 ${ }^{13}$; en éste, la Neisseria meningitidis está sujeta a vigilancia de laboratorio y de resistencia a antimicrobianos. El Nivel Local notifica a la Autoridad Sanitaria Regional, y ésta, al Departamento de Epidemiología del MINSAL. A su vez reporta y deriva las muestras al Laboratorio Nacional de Referencia del Instituto de Salud Pública de Chile (ISP), responsable de confirmar 
la presencia del agente infeccioso, seroagrupar toda muestra proveniente de un caso sospechoso de infección meningocócica y realizar los estudios de susceptibilidad y genéticos. El Laboratorio de Referencia participa en el Sistema de Redes de Vigilancia de los Agentes Responsables de Neumonías y Meningitis Bacterianas (SIREVA II) coordinada por OPS, responsable de la estandarización y control de calidad de las metodologías diagnósticas en nuestra región.

\section{Diagnóstico nivel local}

Frente a un caso sospechoso, definido como todo paciente que presente fiebre súbita mayor o igual de $38^{\circ} \mathrm{C}$ y cefalea asociado a uno o más síntomas y signos que hagan sospechar un síndrome meníngeo: (a) alteración de conciencia como somnolencia, confusión, letargia, estupor o coma (definido por Escala Glasgow); (b) rigidez de nuca; (c) signos de irritación meníngea (Kerning, Bruzinky). Puede presentar además alguno de los siguientes síntomas o signos: convulsiones, rash purpúrico o petequial, náuseas, vómitos ${ }^{14}$. El laboratorio local debe realizar la tinción de Gram y microscopia directa que determina la presencia de diplococos Gram (-), el estudio citoquímico del líquido céfalo raquídeo (LCR), el cultivo de LCR o sangre (gold estándar) que permite el aislamiento del agente infeccioso. Si a las $24 \mathrm{~h}$ los cultivos no presentan desarrollo bacteriano, frente a un caso sospechoso, el sistema de vigilancia incluye la derivación de la muestra de LCR al Laboratorio de Referencia, bajo estrictas condiciones de bioseguridad, para realizar detección molecular por la técnica de reacción en cadena de polimerasa en tiempo real (PCR-RT) ${ }^{15,16}$.

\section{Diagnóstico definitivo: laboratorio de referencia}

En este nivel se realiza la confirmación microbiológica de identificación bacteriana y la caracterización serológica (seroagrupación) utilizando los estándares y control de calidad de la red SIREVA ${ }^{17}$. Para ello se realiza una batería bioquímica en la que se observa la producción de ácido a partir de diferentes carbohidratos y se determina el grupo serológico mediante la técnica de aglutinación en lámina ${ }^{18}$. El caso confirmado se define como caso sospechoso con confirmación de cultivo microbiológico o nexo epidemiológico con un caso confirmado, en ausencia de cultivo se considera la confirmación clínica ${ }^{15}$.

Para efectos de subtipificación molecular se realiza electroforesis de campo pulsado (PFGE) y secuenciamiento genético de multilocus (MLST); este último identifica los distintos linajes genéticos que componen la estructura poblacional de patógenos distribuidos globalmente. Se basa en el análisis de genes metabólicos conservados (house keeping genes) previamente definidos y que para el estudio de Neisseria meningitidis incluye los genes abcZ, adk, aroE, fumC, gdh, pdhC, pgm. Estos fragmentos son amplificados y secuenciados siguiendo el protocolo de la base de datos internacional MLST de la Universidad de Oxford ${ }^{19,20}$. Las secuencias de los fragmentos internos de los siete genes son ingresadas a la base de datos MLST para la determinación del perfil alélico y su correspondiente tipo de secuencia (ST). Los diversos STs, según sus relaciones genéticas, son agrupados en distintos complejos clonales (CC).

Mediante la concentración inhibitoria mínima (CIM), según estándares del Clinical and Laboratory Standards Institute (CLSI) vigentes, se determina la susceptibilidad de la bacteria frente a penicilina, ceftriaxona, rifampicina, cloranfenicol y ciprofloxacino ${ }^{21}$.

Las fuentes de información utilizadas son las notificaciones a través del sistema ENO y vigilancia de laboratorio. Para identificar los casos fallecidos, se utilizó el registro del Departamento de Estadísticas en Salud (DEIS-MINSAL).

\section{Plan de análisis}

Se realizó un estudio observacional de las bases de datos ya señaladas y se incluyeron los casos notificados entre la semana epidemiológica (SE) 1 y 52 del año 2012. Los datos fueron tabulados en una tabla Excel y analizados con el software estadístico STATA 11.1. Las variables fueron codificadas y recodificadas según las necesidades del estudio.

Se utilizaron promedios y medianas como medidas de tendencia central y porcentajes de frecuencias. Para probar normalidad, se utilizó la prueba Shapiro-Wilk. Para comparar y analizar, se utilizó el Test Wilcoxon Rank-sum y el Test $\chi^{2}$. Se consideró un $\alpha=0,05$ para obtener significancia estadística.

\section{Resultados}

\section{Epidemiología EMI 2012}

Entre la SE 1 y 52 de 2012, se confirmaron 133 casos de EMI, número superior en $30,7 \%$ a lo esperado según la mediana quinquenal $(101 \mathrm{ca}-$ 
sos), y mayor a lo observado en el mismo período de 2011 (73 casos). Ochenta y siete coma nueve por ciento (117/133) de casos se confirmó por el Laboratorio de Referencia del ISP, 103 de ellos fueron seroagrupados $(88 \%, 103 / 117)$. De éstos, $36,9 \%$ (38/103), correspondieron al serogrupo B y $58,2 \%(60 / 103)$ al serogrupo W135. Esto refleja un cambio en la distribución de los serogrupos que se presentaba en el país, marcado por el incremento del serogrupo W135 desde el año 2010 (Figura 1).
El mayor número de casos de EMI cuyas cepas fueron seroagrupadas, se registraron en las Regiones de Valparaíso y Metropolitana (RM). El aumento inusitado de casos en ambas regiones se debió a distintos serogrupos. En la primera, el serogrupo predominante fue el B y en la RM, el W135 (Figura 2).

Los casos de Neisseria meningitidis W135 se presentaron en 8 regiones del país, con una mayor proporción en la RM (80\% (48/60)) (Figura 3). De

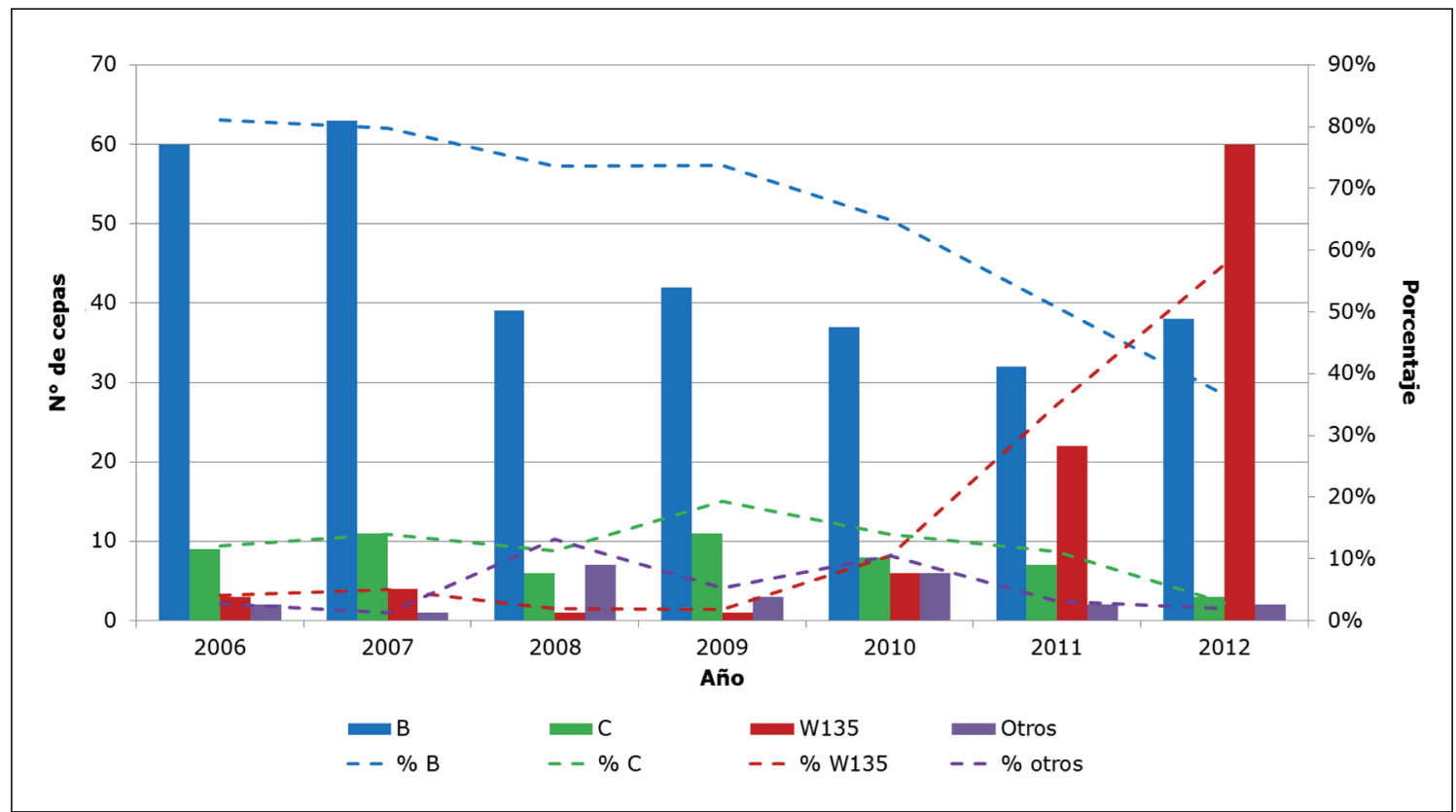

Figura 1. Distribución de casos y porcentaje de Neisseria meningitidis por serogrupo, según año de incidencia. Chile 20062012. Los serogrupos Y, 29E: NT: P1.9, y cepas autoaglutinables se clasificaron como "otros". Fuente: Laboratorio de Referencia de Meningitis Bacteriana. Instituto de Salud Pública de Chile.

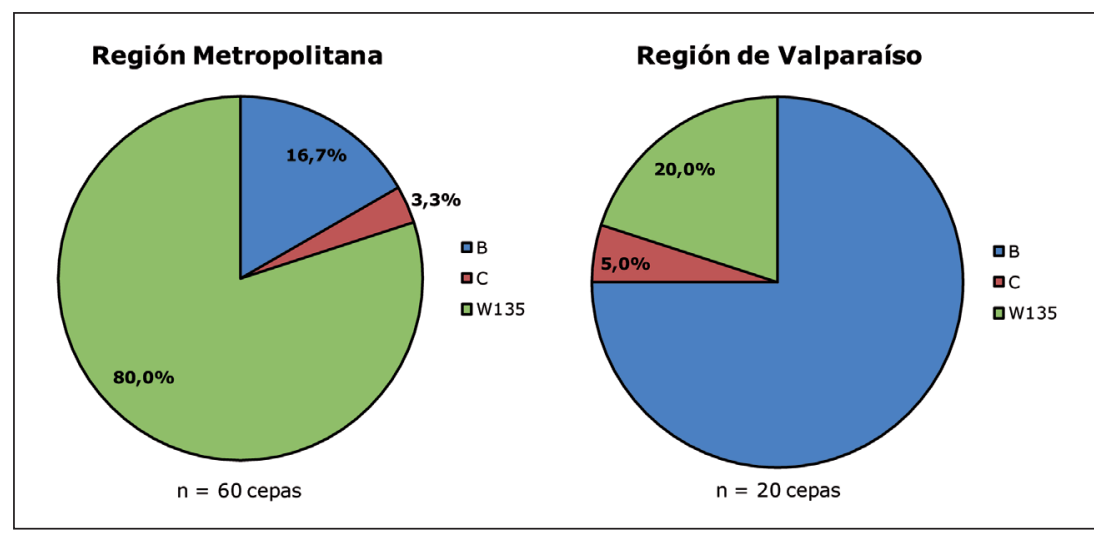

Figura 2. Distribución de casos de Neisseria meningitidis por serogrupo en las regiones Valparaíso y Metropolitana. Chile 2012*. Fuente: Laboratorio de Referencia de Meningitis Bacteriana. Instituto de Salud Pública de Chile. 
los casos registrados a nivel nacional, 25\% (15/60) se presentó en niños menores de 1 año, $21,7 \%$ $(13 / 60)$ en niños de 1 a 4 años y $10 \%(6 / 60)$ en los 65 años y más.

La comparación de las características clínicas de la EMI W135 con los demás serogrupos, permitió identificar que la sepsis o meningococcemia fue más frecuente en los casos W135 que en los otros serogrupos (OR: 5,8; IC: 2,25-15,37). La letalidad de los casos confirmados W135 fue de $26,7 \%$, en

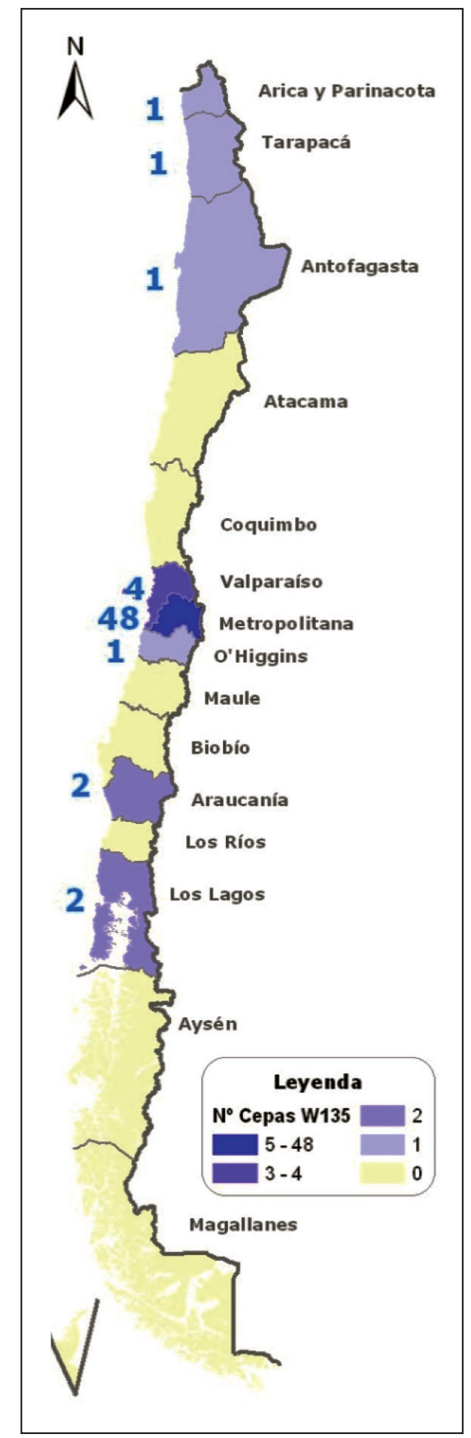

Figura 3. Distribución por región de casos de Neisseria meningitidis W135. Chile 2012. Fuente: Laboratorio de Referencia de Meningitis Bacteriana. Instituto de Salud Pública de Chile. cambio en los casos por otros serogrupos fue de 13,9\% (Tabla 1).

Los resultados del análisis de susceptibilidad in vitro a antimicrobianos de las cepas confirmadas de Neisseria meningitidis serogrupo W135 indican que $16,7 \%$ (10/60) presentaron sensibilidad intermedia a penicilina. Ciento por ciento $(60 / 60)$ fueron sensibles a rifampicina, ciprofloxacino y ceftriaxona (Tabla 2).

Se realizó el análisis de subtipificación genética de 46 de las cepas de Neisseria meningitis W135 del año 2012 (85,2\%); al igual que las cepas de este serogrupo de los años 2010 (6 cepas) y 2011 (22 cepas). Los subtipos genéticos observados con mayor frecuencia en todo el período fueron, en orden de frecuencia, Cl-Nm-Spe-031 (44,6\%, $33 / 74)$, Cl-Nm-Spe-030 (23\%, 17/74) y Cl-NmSpe-046 $(13,5 \%, 10 / 74)$. El año 2012 se observó un aumento en el número de cepas correspondientes al clon Cl-Nm-Spe-031, el cual representó 54,3\% (25/46) de las cepas de ese año. Se realizó el estudio de MLST de 35 cepas, 7 de ellas pertenecientes al año 2012, determinando que 62,9\% (22/35) correspondían a ST11 (Figura 4).

Se analizó la presentación clínica de los casos W135 de acuerdo a los subtipos genéticos identificados, no presentando diferencias significativas.

\section{Discusión}

En Chile, la vigilancia activa de EMI se encuentra amparada en el Decreto 158, de alcance universal y que obliga a notificarla en forma inmediata, permitiendo monitorear y generar alertas. Si agregamos el valioso aporte del laboratorio que estudia los serogrupos responsables de EMI con genotipos específicos, esto conduce a un mejor conocimiento de los cambios y tendencias de la enfermedad en el tiempo, ajustando de esta forma las recomendaciones y estrategias de prevención y control a través de la vacunación u otras intervenciones.

Para comprender la emergencia de la cepa W135 en Chile, en un momento en el que se alcanzaban las tasas de incidencia más bajas $(0,4$ por 100.000 habitantes), fue necesario ubicarnos en el contexto global. Las cepas W135 pertenecientes al complejo clonal ST-11 se han asociado a un brote internacional entre los peregrinos del Hajj en el 2000 y 2001, primer gran brote reportado relacio- 
Enfermedad Meningocócica Invasora W135 en Chile 2012 - M. T. Valenzuela et al

Tabla 1. Características generales, demográficas y clínicas de los casos de EM, según serogrupo. Chile 2012

\begin{tabular}{|c|c|c|c|c|c|c|}
\hline \multirow[t]{2}{*}{ Característica } & \multicolumn{4}{|c|}{ Confirmados por cultivo } & \multirow[t]{2}{*}{$\mathbf{p}$} & \multirow[t]{2}{*}{ OR (95\% IC) } \\
\hline & W135 & $n=60$ & no W135 & $n=43$ & & \\
\hline Sexo masculino & $37 / 60$ & $(61,7 \%)$ & $25 / 43$ & $(58,1 \%)$ & 0,718 & \\
\hline Edad en años (mediana, LS y LI) & 8 & $(0-93)$ & 5 & $(0-74)$ & $0,467^{*}$ & \\
\hline Residencia Región Metropolitana & $48 / 60$ & $(80 \%)$ & $12 / 43$ & $(27,9 \%)$ & $<0,001 * *$ & $10,3 \quad(3,78-28,79)$ \\
\hline Residencia no Región Metropolitana & $12 / 60$ & $(20 \%)$ & $31 / 43$ & $(72,1 \%)$ & $<0,001^{* *}$ & $0,09(0,03-0,26)$ \\
\hline Sepsis (meningococcemia) A39.2 & $40 / 60$ & $(66,7 \%)$ & $11 / 43$ & $(25,6 \%)$ & $<0,001 * *$ & $5,80(2,25-15,37)$ \\
\hline Meningitis A39.0 & $13 / 60$ & $(21,7 \%)$ & $29 / 43$ & $(67,4 \%)$ & $<0,001 * *$ & $0,13(0,05-0,35)$ \\
\hline $\begin{array}{l}\text { Meningococcemia no especificada } \\
\text { (bacteremia) A39.4 }\end{array}$ & $1 / 60$ & $(1,7 \%)$ & 0 & & $0,39 * *$ & \\
\hline Otras infecciones meningocócicas A39.8 & $3 / 60$ & $(5,0 \%)$ & $1 / 43$ & $(2,3 \%)$ & $0,48^{* *}$ & \\
\hline SD Waterhouse-Friderichsen A39.1 & $1 / 60$ & $(1,7 \%)$ & $1 / 43$ & $(2,3 \%)$ & $0,81 * *$ & \\
\hline $\begin{array}{l}\text { Infección meningocócica no especificada } \\
\text { A39.9 }\end{array}$ & $2 / 60$ & $(3,3 \%)$ & $1 / 43$ & $(2,3 \%)$ & $0,76^{* *}$ & \\
\hline Letalidad & $16 / 60$ & $(26,7 \%)$ & $6 / 43$ & $(13,9 \%)$ & $0,12 * *$ & \\
\hline
\end{tabular}

**Test Wilcoxon-Ranksum: Test no paramétrico para dos muestras, p significativo $<0,005 .{ }^{* * *}$ Test $\chi^{2}$ para variables categóricas, p significativo $<0,005$. Fuente: Departamento de Epidemiología. Ministerio de Salud de Chile. Laboratorio de Referencia de Meningitis. Instituto de Salud Pública de Chile.

Tabla 2. Análisis de susceptibilidad in vitro de cepas Neisseria meningitidis. Chile 2012

\begin{tabular}{|llcc|}
\hline Antimicrobiano & W135 & no W135 & \\
Penicilina & $\begin{array}{l}\text { Sensible } \\
\text { Intermedia }\end{array}$ & $50 / 60$ & $7 / 43$ \\
& $60 / 60$ & $36 / 43$ \\
Rifampicina & $60 / 60$ & $43 / 43$ & \\
Ciprofloxacino & $60 / 60$ & $43 / 43$ & \\
Ceftriaxona & $60 / 60$ & $43 / 43$ & \\
\hline
\end{tabular}

Fuente: Laboratorio de Referencia de Meningitis Bacteriana. Instituto de Salud Pública de Chile.

nado al serogrupo W135 que registró más de 400 casos en todo el mundo al retornar los peregrinos, tanto en ellos como en sus contactos 22 .

La EMI tiene un comportamiento difícil de predecir, para dilucidarlo la ciencia ha dedicado esfuerzos importantes para conocer el origen de las cepas e investigar la relación con los complejos clonales mayores. Para ello se realizó subtipificación genotípica y fenotípica en algunos aislamientos, tanto del brote como provenientes de todo el mundo desde el año 1970 hasta el año 2000. Todos los aislamientos provenientes del brote antes señalado eran miembros de un clon único del tipo electroforético hipervirulento 37, el (W) ET-37. Se denomina clon hipervirulento a cepas estrechamente relacionadas (clones o linajes), particularmente asociados con brotes de enfermedad o cepas con mayor capacidad de producir enferme$\mathrm{dad}^{23}$. Este brote ha sido causado por la expansión del clon (W)ET-37, que ha estado en circulación al menos desde 1970. El clon identificado mediante el secuenciamiento genético por MLST fue ST11, asociado con cepas del complejo (W)ET-37, reconocido como hiperinvasivo, causante de meningococcemia de alta severidad y letalidad ${ }^{23}$.

En Brasil, desde enero de 2003 a diciembre de 2005, se reportaron 493 casos y una incidencia de 1,54 casos por 100 mil habitantes. Se observó por primera vez un incremento de los casos de W135 en Rio do Sul (17\%), lo que generó un aumento de la incidencia en $17,8 \%$ respecto de años anteriores. Se identificaron 32 diferentes ST, la mayor prevalencia correspondió a los linajes W135; $\mathrm{P}_{1,5,2: \mathrm{ST}-11}{ }^{24}$. 


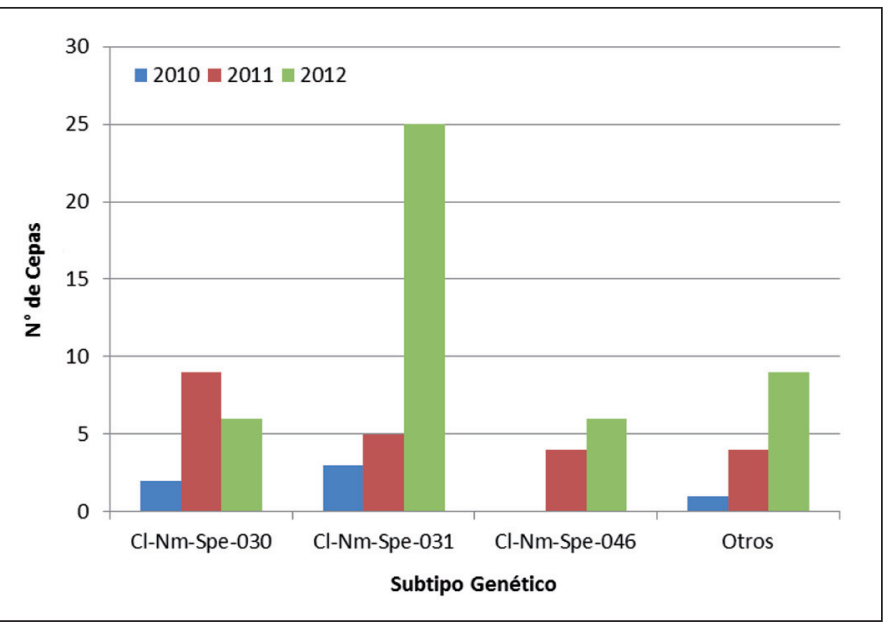

Figura 4. Subtipos genéticos identificados en cepas de Neisseria meningitidis W135. Chile 2012-2013. Fuente: Subdepartamento de genética molecular. Instituto de Salud Pública de Chile.
En Argentina, la tasa de incidencia de la EMI es 0,6 casos por 100 mil habitantes. El serogrupo B había sido el más frecuente hasta el año 2006, alrededor de $65 \%$. A contar de ese año apareció una nueva situación, con una proporción similar de aislamientos del serogrupo C, Y, W135 (7\% en cada uno), que evolucionó hasta $11,4 \%, 6,8 \%$ y $13 \%$, respectivamente, durante el 2007. El aumento de la cepa W135 comenzó a ocurrir en la provincia de Misiones, que limita al norte con Brasil. En el 2008, el serogrupo W135 aumentó dramáticamente, alcanzando $27,7 \%$ y en el 2010, 50\%, desplazando al serogrupo B. La mayoría de los casos (63\%) aparecieron en los niños menores de 9 años, con $28 \%$ en niños menores de 1 año $^{25}$. El sistema de vigilancia epidemiológica en Argentina difiere del sistema chileno, ellos comienzan la investigación a partir del laboratorio, frente al aislamiento de Neisseria meningitidis a partir de un fluido estéril y en forma retrospectiva realizan la investigación clínica. Por otra parte, reconocen que el número de cepas de meningococo recibidas en el Laboratorio Nacional de Referencia, Dr. Carlos Malbrán, representa cerca de $50 \%$ de los casos de EMI en el país. (Comunicación personal de Dra. Sandra Sagradini, Responsable del Programa Nacional de Meningitis del Ministerio de Salud de Argentina).

En Miami, Florida, entre diciembre del año 2008 y abril de 2009 se observó un aumento de EMI por serogrupo W135 en el Sur de Florida, reportándose 14 casos. Nueve de ellos con un patrón por electroforesis de campo pulsado (PFGE) indistinguible. La presentación clínica predo- minante fue el síndrome bacterémico (85,7\%), con una letalidad de $28,6 \%{ }^{26}$. Molecularmente se caracterizaron 7 de los aislados mediante PCR y MLST, evidenciando que las cepas pertenecían al clon ST-11 del complejo clonal 37, íntimamente relacionado al brote de los peregrinos de la $\mathrm{Meca}^{22}$. Cuando ocurre la emergencia de esta cepa en Florida, la incidencia anual de infección meningocócica había declinado gradualmente desde 1,2 a 0,4 casos por 100 mil habitantes entre los años 1997 al 2006, respectivamente ${ }^{26}$.

Con lo anteriormente expuesto, es evidente que Chile no ha quedado indemne a la llegada de la cepa emergente W135, clon ST-11 hipervirulento. En general se observa que los países que han reportado la presencia de esta cepa como principal causante de los casos de EMI, se encontraban en una situación epidemiológica de descenso de la incidencia, con tasas inferiores a 0,8 por 100.000 habitantes.

Entre las lecciones aprendidas destaca que la mejor forma de monitorear esta grave enferme$\mathrm{dad}$, que genera una alta letalidad, es la vigilancia epidemiológica integrada, universal, con un fuerte componente epidemiológico, clínico y de laboratorio. Este último componente es el que nos ha permitido confirmar la emergencia de una cepa que ya circulaba desde hace años en nuestra región, comprendiendo el dinamismo de los serogrupos.

Quedan varios aspectos por aclarar, entre ellos, las condiciones del huésped, la importancia de la portación faríngea en el incremento de la incidencia; en cuanto al agente, profundizar si la 
Enfermedad Meningocócica Invasora W135 en Chile 2012 - M. T. Valenzuela et al

hipervirulencia está relacionada con el clon ST-11 o con la cepa W135. Sin embargo, lo más relevante es que frente al aumento inusitado de casos por la cepa W135, el Ministerio de Salud de Chile realizó una intervención activa y oportuna, desarrollando un plan estratégico para abordar la situación a través del Plan de Acción W-135.

Agradecimientos: A todo el personal de salud que colabora en la red de vigilancia integrada en Chile.

\section{Referencias}

1. Meningococcal: Meningococcal Meningitis And Septicaemia Notifiable. En: Salisbury D, Ramsay M, Noakes $\mathrm{K}$, eds. Immunisation against infectious disease. 3era ed. ed. Londres: The Stationery Office; 2013. p. 235-59.

2. Instituto de Salud Pública. Vigilancia de Laboratorio de enfermedad invasora Neisseria meningitidis. Laboratorio de Referencia 2006-11. Boletin Instituto de Salud Pública de Chile 2012; 2 (2): 1-18. Disponible en: http://www. ispch.cl/sites/default/files/Bolet\%C3\%ADn\%20Neisseria\%20Meningitidis\%2026\%20marzo.pdf. [Consultado el 23 de enero de 2013].

3. Organización Mundial de la Salud. Global Alert and Response (GAR): Impact of the problem 2013; Disponible en: http://www.who.int/csr/disease/meningococcal/ impact/en/index.html. [Consultado el 30 de enero de 2013].

4. Organización Mundial de la Salud. meningococcal vaccines: WHO position paper, november 2011 Wkly. Epidemiol Rec 2011; 86 (47): 521-40. Disponible en: http://www.who.int/wer/2011/wer8647.pdf. [Consultado el 11 de enero de 2013].

5. Organización Mundial de la Salud. The immunological basis for immunization series: module 15: meningococcal disease. Geneva: World Health Organization; 2010. p. 81 Disponible en: http://whqlibdoc.who.int/publications/2010/9789241599849_eng.pdf. [Consultado el 4 de enero de 2013].

6. Brooks R, Woods CW, Benjamin DK, Rosenstein NE. Increased case-fatality rate associated with outbreaks of Neisseria meningitidis infection, compared with sporadic meningococcal disease, in the United States, 1994-2002. Clin Infect Dis 2006; 43 (1): 49-54.

7. Oostenbrink R, Maas M, Moons KGM, Moll HA. Sequelae after bacterial meningitis in childhood. Scand J Infect Dis 2002; 34 (5): 379-82.

8. Castillo L, Maldonado A, García J, Silva W, Ulloa MT,
Valenzuela MT, et al. Caracterización de Neisseria meningitidis aislada de infecciones sistémicas: Chile, 19921993. Rev Med Chile 1994; 122 (7): 760-67.

9. Ministerio de Salud de Chile. Control de la Enfermedad Meningocócica. Guía de actualización 1995. Santiago de Chile: MINSAL; 1995. 81 p.

10. Ministerio de Salud de Chile. Departamento Epidemiología. Unidad de Vigilancia. DIPLAS. Informe de situación epidemiológica Enfermedad Meningocócica en Chile, año 2012. Actualizado al 16/11/2012. Santiago de Chile: MINSAL; 1995.

11. Instituto Nacional de Estadísticas. Resultados preliminares Censo de población y vivienda 2012. Santiago de Chile: Instituto Nacional de Estadísticas; 2012. Disponible en: http://www.censo.cl/2012/08/resultados_preliminares_censo_2012.pdf [Consultado el 23 de enero de 2013].

12. Instituto Nacional de Estadísticas. Chile: Proyecciones y Estimaciones de Población 1990-2020 - País y Regiones En: Demografía Dd, ed. Santiago de Chile: Instituto Nacional de Estadísticas; 2012: p. 86. Disponible en: http:// www.ine.cl/canales/chile_estadistico/demografia_y_vitales/demo_y_vita.php [Consultado el 18 de enero de 2013].

13. Ministerio de Salud de Chile. Reglamento sobre notificación de enfermedades transmisibles de declaración obligatoria. Decreto Supremo 158/04. Vol Decreto Supremo 158/04. Santiago de Chile; 2005.

14. Ministerio de Salud de Chile, Subsecretaria de Salud Pública. Ord. B51/841. Refuerza Vigilancia Meningitis bacterianas y Enfermedad meningocócica 2012. Santiago de Chile; 2012.

15. Ministerio de Salud de Chile. Circular B51 No 50 . Circular de vigilancia epidemiológica de meningitis bacterianas. Santiago de Chile; 2011.

16. Ministerio de Salud de Chile. Circular B51 No 09. Vigilancia epidemiológica y medidas de control de enfermedad meningocócica (A39) 2009. Santiago de Chile; 2009.

17. Ibarz A, Lemos A, Gorla M, Brandileone M. Diagnóstico de laboratorio de las meningitis bacterianas causadas por Neisseria meningitidis: Manual de procedimientos de laboratorio de la red SIREVA II. Sao Paulo, Brasil: Organización Panamericana de la Salud. 2011. Disponible en: http://new.paho.org/hq/index.php?option=com docman\&task=doc_download\&gid $=17866 \&$ Itemid $=\&$ lang=es [Consultado el 10 de enero de 2013].

18. Taha MK. Simultaneous approach for nonculture PCRbased identification and serogroup prediction of Neisseria meningitidis. J Clin Microbiol 2000; 38 (2): 855-57.

19. Maiden MC, Bygraves JA, Feil E, Morelli G, Russell JE, Urwin R, et al. Multilocus sequence typing: a portable 
approach to the identification of clones within populations of pathogenic microorganisms. Pro Natl Acad Sci USA 1998; 95 (6): 3140-45.

20. Jolley K. Neisseria Multi Locus Sequence Typing website. Oxford: University of Oxford; 2012. 2012; Disponible en: http://pubmlst.org/neisseria/ [Consultado el 30 de enero de 2013].

21. CLSI. Performance Standards for Antimicrobial Susceptibility Testing; Twenty-Two Informational Supplement. Vol M100-S22. Pennsylvania, USA: Clinical and Laboratory Standards Institute; 2012. p. 184.

22. Lingappa JR, Al-Rabeah AM, Hajjeh R, Mustafa T, Fatani A, Al-Bassam T, et al. Serogroup W-135 meningococcal disease during the Hajj, 2000. Emerg Infect Dis 2003; 9 (6): 665-71.

23. Mayer LW, Reeves MW, Al-Hamdan N, Sacchi CT, Taha MK, Ajello GW, et al. Outbreak of W135 meningococcal disease in 2000: not emergence of a new W135 strain but clonal expansion within the electophoretic type-37 complex. J Infect Dis 2002; 185 (11): 1596-605.

24. Weidlich L, Baethgen LF, Mayer LW, Moraes C, Klein CC, Nunes LS, et al. High prevalence of Neisseria meningitidis hypervirulent lineages and emergence of W135: P1.5,2:ST-11 clone in Southern Brazil. J Infect 2008; 57 (4): 324-31.

25. Efron AM, Sorhouet C, Salcedo C, Abad R, Regueira M, Vázquez JA. W135 Invasive Meningococcal Strains Spreading in South America: Significant Increase in Incidence Rate in Argentina. J Clin Microbiol 2009; 47 (6): 1979-80.

26. Doyle TJ, Mejia-Echeverry A, Fiorella P, Leguen F, Livengood J, Kay R, et al. Cluster of serogroup W135 meningococci, southeastern Florida, 2008-2009. Emerg Infect Dis 2010; 16 (1): 113-5. 